\title{
Coevolution of Interorganizational Psychological Contract and Interorganizational Relationship: A Case Study of Manufacturing Company in China
}

\author{
Wei He \\ School of Business Administration, Jiangxi University of Finance and Economics, No. 169, East Shuanggang Road, Changbei, \\ Nanchang, Jiangxi 330013, China \\ Correspondence should be addressed to Wei He; 04hrirene@163.com
}

Received 31 December 2016; Revised 20 June 2017; Accepted 10 August 2017; Published 17 October 2017

Academic Editor: Paolo Renna

Copyright (C) 2017 Wei He. This is an open access article distributed under the Creative Commons Attribution License, which permits unrestricted use, distribution, and reproduction in any medium, provided the original work is properly cited.

\begin{abstract}
As the belief of the beholder in an exchange about the obligations that another party should have, interorganizational psychological contract (IPC) from a micro perspective provides a new angle to study interorganizational relationship (IOR). This paper studies the interrelation and coevolution of IORs and IPCs by building a system dynamics (SD) model. Firstly based on the structural analysis of the interrelations of IPC and IOR, this paper builds the qualitative causal loop diagram of the interrelations. Based on investigation of 55 manufacturing enterprises in China we further draw the stock and flow diagram. Then we apply the data of Jiangxi Motors Co., Ltd., to simulate the model. The results reveal the development and evolution of IORs and IPCs and their interrelations. Furthermore, the sensitivity analysis is conducted and the influences of trust on IORs and IPCs are discussed. Finally managerial implications and some recommendations are provided for the decision-making of developing IORs.
\end{abstract}

\section{Introduction}

To keep sustainable growth, external linkage is deemed as important path [1]. Through cooperation, enterprises can obtain important resources from their partners, which contribute to the development of the enterprises [2]. Furthermore, Sánchez-González and Herrera (2015) emphasized the importance of external cooperation for overcoming the obstacle of innovation [3]. Aware of the benefits external linkage brings to them, nowadays enterprises attach greater importance to the establishment and maintenance of IOR, namely, the relatively enduring transactions, flows, and linkages that occur among or between an organization and one or more organizations in its environment [4].

IORs management is a hot issue attracting many attentions in recent years [5]. Based on the analysis of alliance management, Ireland et al. (2002) summarized three theoretical frames to understand IORs and strategic alliances: transactions cost, social network theory, and the resourcebased view [6]. However, all these frames can not well explain how the subjective understanding of individual enterprise about the IOR can influence the development of IOR. In another word, these frames cannot give good explanations to how IORs develop from a microcosmic perspective. To address this problem, this paper discusses the coevolution of IORs and IPC to reveal the black box of the development of IORs. In fact, it is well acknowledged that IORs and IPCs are closely related. Both IPCs and IORs are grounded in the social exchange theory and built based on expectations and obligations. They are naturally connected. But how they interplay and coevolve with each other is a question no one addresses. In fact, discussing the coevolution of IPCs and IORs is important. It can contribute to better understanding of the development of IORs and hopefully providing recommendations for the decision-making with regard to IORs. In this paper we choose a system-analysis model to study the issue. Estimating the influencing mechanism between IORs and IPCs is a complex problem due to the presence of multiple technological limitations, feedback processes among subsystems, and various kinds of delays. So system dynamics are a suitable approach to such a complex model.

In conclusion, IPC provides a micro angle to study IOR. But until now there is rare study focusing on the 
interaction of IPC and IOR. This paper tries to discuss the coevolution of IORs and IPCs to reveal the black box of the interplaying between IPC and IOR and thereby provide valuable suggestions for the development of IORs. This paper proceeds as follows: in the second section, we give a literature review on current studies; in the third section, we make a structural analysis on the relationship between IOR and IPC; in the fourth section, we build a SD model to analyze the coevolution of IORs and IPCs and apply the data of Jiangxi Motors Co. Ltd., to simulate the model; in the fifth section, we draw conclusions based on the simulation results and give suggestions for related decision-making activities.

\section{Literature Review}

Entering the 21st century, with the booming of the IOR studies, a hot issue in this field which has attracted many attentions is the discussion on the relationship between the IOR and IPC. IOR is the relatively enduring transactions, flows, and linkages that occur among or between an organization and one or more organizations in its environment [4]. This kind of relationship is built on self-discipline, trust, and interdependence. It is neither the same with the pure transactional exchange in market nor similar to the clear authority in an organization. The studies on IOR can be traced back to 1960 s. After more than 50 years' development, the study of IOR has become an important branch in organizational theory. There are mainly three theory sources to study IORS. The first is the transactional cost perspective: they understood the IOR as way to reduce the uncertainty caused by market failure and the high cost related to hierarchical system [711]. The second is the resource-based perspective: they posited that it was the resources and the core resources in particular that shaped the IOR [12]. And IOR is nothing more than a way for organizations to get access to the resources they need $[13,14]$. The third is the social exchange perspective: they posited that an IOR was fundamentally a process of social exchange [15]. Because of the difficult evaluation of social exchange rewards and the future orientation of social exchange, it requires the use of relational attitudes and behaviors.

Psychological contract is widely thought as the personal understanding of the exchange relationship between employees and employers [16]. In fact, as a kind of contract, psychological contract widely exist in all kinds of exchange relationships [17-20]. It is posited that the expectations and obligations that drive future action within the employment relationship can also be modeled in the organization-toorganization relationship under the auspices of psychological contract. So IPC is the belief of the beholder in the transactional exchange about the obligations another party should have. From the definition, we can know firstly IPC is a perception of mutuality and in an IPC an organization defines their obligations as well as the other parties' obligations within the relationship. Psychological contract therefore exists in IOR when (1) one party believes a promise of future return has been made; (2) a contribution has been given that results in obligations being created to provide future benefit [21]. So clearly IPC is built on promissory-based obligations. On the other hand, according to Grönroos (1990), the establishment of relationships involves the giving of promises, whereas the maintenance of relationships comprises the fulfillment of these promises [22]. The enhancement of relationships can only be accomplished with a new set of promises that are totally contingent upon the fulfillment of earlier promises. This argument appears to be highly congruous with the conceptualization of psychological contract and both constructs are grounded in social exchange theory, which is underpinned by the norm of reciprocity and involved in the perceived obligations between actors within any particular social system [23]. Hence, it is contended that IPC and IOR are closely related [24-27]. There are some studies focusing on discussing the relationship between IPC and IORs.

Kingshott (2002) provided a profound insight in the relationship between IPC and IORs [24]. He pointed out that the psychological contract develops with the growth of IORs. On the one hand, psychological contract is the expectations and obligations based on promises and there are certain relations between IPC and IOR; on the other hand, psychological contract can complement relationship. Gao and Chen (2008) further analyzed how IPC facilitated the formation of IORs [25]. Usually at the beginning of an IOR, enterprises may make all kinds of promises for future exchange. Once these promises are fulfilled during the relationship, it will motivate organizations to make new promises. As such, the IORs can be strengthened with "promise making, promise fulfillment, and new promise making." The evolution of IORs incorporating the promised obligations is no doubt also the formation of psychological contract. Pepur et al. (2013) made an empirical study and the results showed that IOR mainly influenced the quality of IORs through trust, commitment, and cooperation satisfaction [28]. By discussing the origins of expectation and obligation in IPCs, Zhai et al. (2014) explicated the role of fairness as the mapping function in building the psychological contracts [29]. Based on the analysis, they proposed a new framework for analysis of IORs that encompassed simultaneously explicit, normative, and psychological contracts, arguing that the framework would lead to a new research paradigm.

From these studies we can know that IPC develops with IORs. In fact, it influences and at the same time constrains the evolution of IORs. Both IORs and IPC are formed based on social exchange and reciprocity. And promises and expectations are the crucial elements for both IORs and IPC. The same principle that they conform to indicates they are naturally connected to each other. But what is the exact mechanism of the connection? How each dimension of IOR interplays with IPC? What role does trust play in the development of both IPC and IOR? These studies have not provided clear answers to these questions. What is more, the previous studies were mainly based on theoretical analysis. There is a lack of quantitative evidence to clarify the "how" question. This paper addresses these questions by establishing a SD model to quantitatively test the relationship between IPCs and IORs. The findings are hoped for to add more understanding about how IORs develop from the psychological contract perspective. 


\section{Structure Analysis of System Dynamics Model}

3.1. System Dynamics Method. SD is an approach to understand the behavior of complex systems over time. Originally the method was developed to help corporate managers to improve the understanding of industrial processes [30]. Now it is an important method to learn and solve problems of complex system. Through modeling feedback structure, one can better understand dynamic complexity that can be found in situations where the same action has different effects in the short and the long run and where an action has one set of consequences locally and a different set of consequences in another part of the system.

It is well recognized that IPCs and IORs are closely connected to each other, which are both grounded in social exchange theory. However, the current studies failed to fully discuss the interplaying relationship between the two concepts. In another word, the conventional literature neither explored the two-way interactions between IPCs and IORs nor noticed both the positive and negative influences of IPC on IORs. Therefore, it is obvious that a good and wellstructured practice is required in order to better understand this relationship. Considering the complex and dynamic nature of the relationship, SD has been used.

3.2. Structure Analysis of the Interrelations between Interorganizational Psychological Contract and Interorganizational Relationship. IPC develops with IORs. Psychological contracts are said to exist when an individual believes that certain promises have been made which result in perceived obligations. On the other hand, Grönroos (1990) pointed out that the establishment of relationships involved the giving of promises, whereas the maintenance of relationships comprised the fulfillment of these promises [22]. Both of them rely on promises and obligations and are grounded in social exchange theory. So it is easily deduced that IPC and IORs should have certain relation.

According to Pepur et al's (2013) study, IPC mainly influences the quality of IORs through trust, commitment, and cooperation satisfaction [28]. Based on the study of interactive activities and socialization of cooperation, Kingshott (2006) analyzed the three ways which IPCs may influence trust and commitment [31]. Firstly, what distinguishes psychological contract from other forms of agreement is that they are highly perceptual and idiosyncratic in nature. Hence, on the one hand, the perceived obligations will act as the glue that binds parties. On the other hand, fulfillment of these obligations will increase the level of reliance upon the other party. Secondly, sending signals and communicating are essential elements of an IOR [24]. The fulfillment of psychological contract determines the extent of optimism one party holds towards the cooperative relationship. As the obligations are fulfilled, the optimistic signals are sent which influences the expectations for the transaction and therefore influences the IOR. Thirdly, a well-fulfilled psychological contract can contribute to higher cooperation satisfaction. The party will be quite satisfied if its psychological contract is recognized and fulfilled by the other party. Except for these influences, with regard to the development of IORs, opportunistic behaviors are important factor we should take into consideration. As we discussed above, the fulfillment of perceived obligations may increase the reliance on the other party, which may be more likely to bring opportunistic behaviors. As a consequence, the IOR is damaged.

At the same time, with the development of IORs, communication and cooperation are increased and relational norms are formed [32]. Parties' behaviors conform to a set of uniform standards and norms and with the increase of interactions they gradually recognize each other's values. Such recognition among cooperative organizations contributes to better understanding of each other's obligations and responsibilities in the relationship. Hence, such recognition fosters the fulfillment of IPC. On the other hand, IORs naturally have relational properties [33]. With the development of IORs, relational norms are dominant for governing the relationship while the use of formal contract is reduced. The reduced use of formal contract may influence the properties of perceived obligations. In other words, transactional obligations are reduced, which may also influence the fulfillment of IPCs.

Based on the above theoretical analysis, the structure characteristics of the relationship between IORs and IPCs are identified and the influences on IORs and IPCs can be summarized as follows:

(1) There are both positive and negative influences of IPCs on IORs: firstly, because a well-fulfilled IPC can better satisfy the cooperative party, it always leads to high cooperation satisfaction. When both parties are satisfied with each other, they are more willing to cooperate with each other and prolong their relationship. Secondly, a well-fulfilled IPC indicates one party can always fulfills its obligations of the transaction. With the continuous implementation of the obligations, the other party will more trust it and gradually will depend more on it. However, due to the rationality rule, the increase of dependence on one party will easily lead to the opportunistic behaviors of another party. To sum up, IPCs mainly work through cooperation satisfaction to positively influence IORs; at the same time IPCs also work through dependence and opportunistic behaviors to negatively influence IORs.

(2) There are both positive and negative influences of IORs on IPCs: firstly, with the development of IORs, there is more cooperation between the two parties. They have more opportunities to interact with each other and during this process they may intend to recognize each other's values and missions more and form good intentions towards each other. All these contribute to better understanding of each other's obligations in the cooperation and thereby the fulfillment of the IPC. Secondly, if the relationship of both parties develops, when encountering problems parties will depend more on informal ways such as the relational norms rather than formal contract to deal with it. In other words, if the IOR between two parties develops well, there are less detailed terms in the contract and the contract is more incomplete. Hence, 


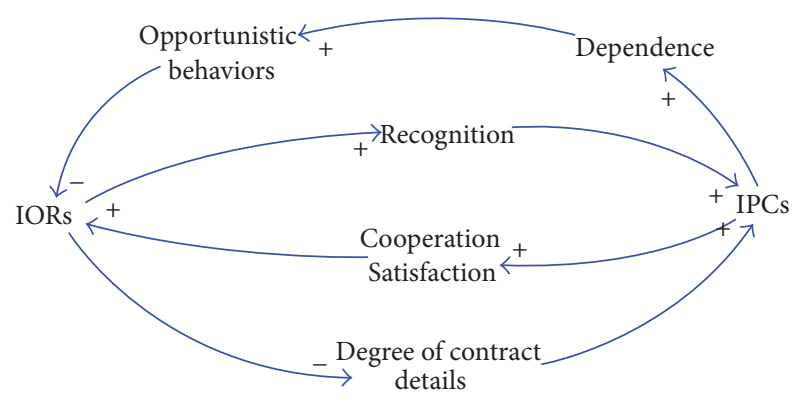

FIgURE 1: Causal loop diagram of interrelations between IORs and IPCs.

a direct outcome is the decrease of perceived transactional obligations in an IPC, which may greatly influence the fulfillment of IPCs. To sum up, IORs mainly work through recognition to positively influence IPCs; at the same time IORs also work through the degree of contract details to negatively influence IPCs.

Based on the above analysis, we can build the qualitative causal loop diagram of the interrelations between IPCs and IORs as shown in Figure 1. Figure 1 mainly reflects the influence paths of IPCs on IORs and the influence paths of IORs on IPCs. The arrows in Figure 1 represent the influential relationship between two factors. The positive sign and negative sign represent the positive and negative correlations, respectively.

3.3. Model Assumptions. This study makes the following basic assumptions in order to quantify the structure model and build complete SD model.

Assumption 1. IPC is divided into transactional psychological contract and relational psychological contract [24]. Thereby IPC is represented by the amount of transactional psychological contract implemented and the amount of relational psychological contract implemented.

Assumption 2. IOR is divided into relationship intensity, relationship length, and relationship justice [34]. Thereby IOR is represented by level of relationship intensity, which is expressed with relationship stock, level of relationship length, and level of relationship justice which is expressed with degree of relationship justice.

\section{Model Simulation}

In this section, we take Jiangxi Motors Co., Ltd., as example to explore the relationship between IPC and IOR.

\subsection{Model Construction}

4.1.1. Investigation and Interviews. Based on the causal loop diagram developed in Section 3, we perform a more detailed quantitative analysis to transform the causal loop diagram to a stock and flow diagram. The first step we take is to determine all the variables needed to describe the system structure.
In the system, IOR and IPC are the two basic elements. According to model Assumptions 1 and 2, IPC can be divided into transactional psychological contract and relational psychological contract [31]. IOR can be divided into relationship intensity, relationship length, and relationship justice [34]. So these five dimensions, respectively, form 5 branches of a rate variable fundamental in-tree model. Correspondingly there are five level variables: relationship stock, relationship length, degree of relationship justice, amount of transactional psychological contract, and amount of relational psychological contract. There are also five rate variables: relationship growth rate, increase of relationship length, changes of relationship justice, changes of transactional psychological contract, and changes of relational psychological contract.

To draw the branches, parameters are extracted partly based on related studies and partly from the structural analysis and interviews we conducted. First we make theoretical analysis of each variable to find out the crucial factors influencing each rate variable and their relationship. Based on it, we conduct interviews with 78 managers in 55 enterprises. All these enterprises are manufacturing companies in Jiangxi province, among which 24 are located in Nanchang city, 9 in Ganzhou city, 11 in Yingtan city, 7 in Fuzhou city, and 4 in Xinyu city. The managers we interviewed are the middle level managers and they are the responsible persons to interact with their partner enterprises. We provide them with semiopen questionnaires which contain the crucial factors we get from theoretical analysis. They are asked to check the importance of these factors and they also can add factors which they think are important. For those factors deriving from the theoretical analysis, the ones which two-thirds of the managers thought are not important and irrelevant are then deleted. The others remain. For those factors added by managers, the ones which two-thirds of the managers thought are important remain. During the process, we also invite 4 management professors of Jiangxi University of Finance and Economics to further discuss these factors remained and their relationship. Then based on it the five rate variable in-tree branches are built.

Next we give specific explanation of how each in-tree branch is built.

Regarding relationship stock, the relationship growth rate is influenced by degree of closeness, degree of reciprocity, and degree of emotion. This can refer to Zhang et al's (2012) study [35]. Then, respectively, degree of closeness is mainly influenced by time and frequencies of communication. Closeness here refers to being near in relationship. According to the interviews of managers, with time going by and more communications the two parties in a relationship can be closer. So degree of closeness is mainly influenced by time and frequencies of communication. Degree of reciprocity is mainly influenced by degree of dependency, degree of trust, and cooperative willingness. Reciprocity here refers to that given or felt by each toward the other in a relationship. According to social exchange theory, interdependency is the basis for reciprocity. So dependency is an important factor influencing degree of reciprocity. Besides, the willingness to cooperate is the precondition for reciprocity. And trust is an important factor which binds the two parties in a relationship 
together. So based on theoretical analysis and the interviews of managers, we propose that degree of reciprocity is mainly influenced by degree of dependency, cooperative willingness, and degree of trust. Here we design degree of trust as an external variable and we will further discuss its influence on IPC and IOR. For the other two factors, we interviewed experienced managers and asked them whether the two factors could be connected with transactional psychological contract or relational psychological contract or both. According to the results of interviews, degree of dependency is directly influenced by the amount of transactional psychological contract implemented. Usually as more transactional psychological contract is implemented, it shows the party has high ability to honor the promises it makes and therefore will increase the other parties' dependency on it. According to the structural analysis and interviews of managers, cooperative willingness is mainly influenced by cooperation satisfaction and the amount of relational psychological contract implemented. The cooperation satisfaction of previous exchange mainly resulting from the fulfillment of transactional expectations will lead to the willingness to conduct next cooperation. And according to managers, the fulfillment of some relational expectations such as providing support to cooperative partners can largely lead to relatively high cooperative willingness. Lastly emotion refers to the affective state in a relationship. On the organizational level, the fulfillment of relational expectations can increase the goodwill between the cooperative parties which results in relatively high degree of emotion. Most interviewed managers also confirm this view.

Regarding relationship length, the increase of relationship length is mainly influenced by switch cost, the amount of proprietary investment, and frequencies of transaction. Relationship length refers to how long a relationship lasts. Usually the difficulty of an organization changing its cooperative partner may influence the length of an IOR. The more difficult it is, the longer the IOR is. And we use switch cost to represent such difficulty. Besides, if an organization is willing to invest in an IOR, the IOR will last longer. So the amount of proprietary investment is important for the relationship length. The importance of the two factors to relationship length is also confirmed by the results of the interviews. And apart from the two factors, the interviews also revealed a third important factor, that is, frequencies of transaction. Transactions are the basis of relationship length. The frequencies of transaction are an important index to indicate how long a relationship lasts. For switch cost, we ask managers whether it can be connected with IPC. And according to the results of the interviews, most managers point out that the amount of relational psychological contract implemented greatly influence the switch cost. For the amount of proprietary investment, in the interviews managers point out that cooperative willingness and opportunism cost can exert influence on it. According to them, the willingness to cooperate provides the potential to invest while the opportunism cost indicates the possibility the proprietary investment may loose the value. And according to the results of the interviews, for opportunism cost, degree of dependency is the most important influencing factor.

Regarding relationship justice, the changes of relationship justice are mainly influenced by degree of distributive justice, degree of procedural justice, and degree of interactional justice. This can refer to [36]. To draw the branch, we ask the managers whether each of the three factors can be connected with IPC. According to them, degree of distributive justice is influenced by the amount of transactional psychological contract implemented because the fulfillment of economic expectations is the foundation of the perception to the fairness of outcomes. Degree of procedural justice is mainly influenced by the amount of relational psychological contract implemented because the fulfillment of social and emotional expectations is regarded as important for people to perceive whether an IOR proceeds fairly or not. Degree of interactional justice is mainly influenced by the amount of relational psychological contract implemented and frequencies of communication. In fact, relational psychological contract mainly involves interactions between two parties and the fulfillment of relational psychological contract indicates the social and interactive promises are honored by parties and the interaction between parties is perceived fair by each party.

Regarding the amount of transactional psychological contract implemented, the changes of transactional psychological contract implemented are mainly influenced by enterprise competency and rate of formal contracting. According to the interviews of managers, enterprise competency is the most important factor influencing the amount of transactional psychological contract implemented. Besides, how many promises are made by signing a formal contract also influence the amount of economic expectations fulfilled. And the higher the intensity, length, and justice of a relationship is, generally the less the rate of formal contract is. This is mainly because all these aspects of an IOR will easily lead to the use of relational contract which may reduce the rate of formal contracting.

Regarding the amount of relational psychological contract implemented, the changes of relational psychological contract implemented are mainly influenced by cooperation factor and degree of recognition. Cooperation factor is the rate of cooperative willingness. Because relational psychological contract is about the intangible and social expectations in an exchange, it is not set on paper. Instead, it deeply exists in both parties' minds and it is driven by the willingness to cooperate. So generally the higher the cooperative willingness is, the more the relational psychological contract is fulfilled. According to the managers, degree of recognition is influenced by degree of match of enterprise value, relationship stock, relationship length, and relationship justice. On the one hand, recognition is influenced by the degree of match of two organizations. The higher the degree of match is, the easier it is to reach recognition. On the other hand, through the development of cooperation and relationship, recognition is easier to form.

4.1.2. Variables and Parameters of System Dynamics Model. Based on the results of above interviews and discussion, we modified the expression of each factor and get the final variables of the SD model. Please see Table 1.

(1) Relationship Growth Rate (RGR) Rate Variable InTree Branch. Relationship growth rate (RGR) describes the 
TABLE 1: Parameter list of SD model of coevolution of IORs and IPCs.

\begin{tabular}{|c|c|c|}
\hline & Variables & Connotation \\
\hline \multirow{5}{*}{ Level variables } & RS (relationship stock) & Level of relationship intensity \\
\hline & RL (relationship length) & Level of relationship length \\
\hline & DRJ (degree of relationship justice) & Level of relationship justice \\
\hline & $\begin{array}{l}\text { ATPCI (amount of transactional } \\
\text { psychological contract implemented) }\end{array}$ & $\begin{array}{l}\text { Fulfillment of transactional psychological } \\
\text { contract }\end{array}$ \\
\hline & $\begin{array}{l}\text { ARPCI (amount of relational } \\
\text { psychological contract implemented) }\end{array}$ & $\begin{array}{l}\text { Fulfillment of relational psychological } \\
\text { contract }\end{array}$ \\
\hline Rate variables & $\begin{array}{l}\text { RGR (relationship growth rate) } \\
\text { IRL (increase of relationship length) } \\
\text { CRJ (changes of relationship justice) } \\
\text { CTPCI (changes of transactional } \\
\text { psychological contract implemented) } \\
\text { CRPCI (changes of relational } \\
\text { psychological contract implemented) }\end{array}$ & $\begin{array}{l}\text { Relationship growth rate } \\
\text { Increase of relationship length } \\
\text { changes of relationship justice } \\
\text { changes of transactional psychological } \\
\text { contract } \\
\text { changes of relational psychological } \\
\text { contract }\end{array}$ \\
\hline \multirow{14}{*}{ Auxiliary variables } & DC (degree of closeness) & Level of closeness \\
\hline & DR (degree of reciprocity) & Level of reciprocity \\
\hline & DE (degree of emotion) & Level of emotion \\
\hline & DD (degree of dependence) & Level of dependence \\
\hline & CW (cooperative willingness) & Level of willingness to cooperate \\
\hline & CS (cooperation satisfaction) & Level of satisfaction to cooperate \\
\hline & SC (switch cost) & Switch cost \\
\hline & OC (opportunistic cost) & Opportunistic cost \\
\hline & API (amount of proprietary investment) & Amount of proprietary investment \\
\hline & DDJ (degree of distributive justice) & Level of distributive justice \\
\hline & DPJ (degree of procedural justice) & Level of procedural justice \\
\hline & DIJ (degree of interactional justice) & Level of interactional justice \\
\hline & DCD (degree of contract details) & Level of contract details \\
\hline & DOR (degree of recognition) & Level of recognition \\
\hline \multirow{4}{*}{ External variables } & DT (degree of trust) & Level of trust \\
\hline & FC (frequencies of communication) & Frequencies of communication \\
\hline & FT (frequencies of transactions) & Frequencies of transactions \\
\hline & EC (enterprise competency) & Level of enterprise competency \\
\hline \multirow[b]{2}{*}{ Constant } & CF (cooperative factor) & Rate of cooperative willingness \\
\hline & $\begin{array}{l}\text { MDEV (match degree of enterprise } \\
\text { values) }\end{array}$ & Level of alignment of enterprise values \\
\hline
\end{tabular}

changes of relationship stock with time (Figure 2). According to Zhang et al. (2012), RGR is influenced by three factors: degree of closeness (DC), degree of reciprocity (DR), and degree of emotion (DE) [35]. In other words, changes in the three aspects can greatly influence RGR. But such changes will not have direct and immediate impact on RGR. Usually there is a delay in time. So we use a delay function to describe RGR. In terms of DC, we argue that, with the accumulation of closeness, above certain point it can have a sudden change. For example, in some manufacturing companies, they need to divide the suppliers into different levels. Usually the companies may decide the levels of the suppliers according to the transaction frequencies and credit. If the transactions with a supplier exceed a certain number, then the level of the supplier can be upgraded. Considering such characteristics, we use step function to describe DC. And similarly step function is also applied to calculate DR and DD. According to the results of the above interviews, DR is jointly influenced by cooperative willingness (CW), degree of dependence (DD) and degree of trust (DT). FC and DT are external variables. We use investigation and interviews to give the values to them. And we process CS similarly. For equations see Appendix B.

(2) Increase of Relationship Length (IRL) Rate Variable In-Tree Branch. Increase of relationship length (IRL) describes the changes of relationship length. According to the results of the above interviews, it is influenced by frequencies of transactions (FT) and the amount of proprietary investment (API) and switch cost (SC). Please see Figure 3. The more the FT is, the longer the relationship lasts. The higher the API is, which indicates the organization is willing to invest in the relationship, usually the longer the relationship lasts. The higher the switch cost is, the less likely the enterprises build new cooperation relationship and the longer the relationship will be.

According to the results of the above interviews, API is influenced by opportunistic cost (OC) and cooperative willingness $(\mathrm{CW})$. The higher the $\mathrm{OC}$ is, which indicates the organization has to deal with the opportunistic behaviors of its partner, the less the willingness for the organization to 


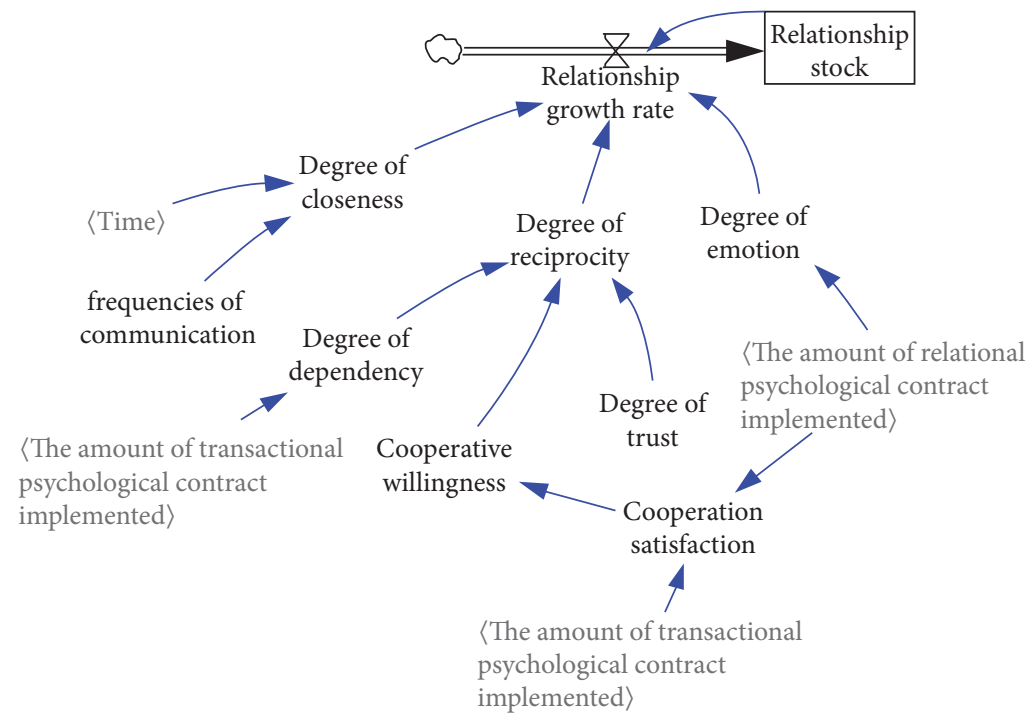

FIGURE 2: Relationship growth rate (RGR) rate variable in-tree branch.

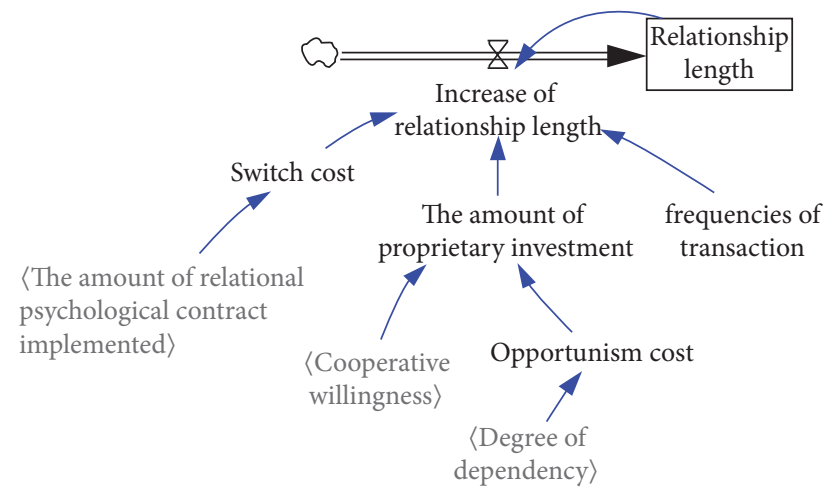

FIGURE 3: Increase of relationship length (IRL) rate variable in-tree branch.

cooperate with and invest in the relationship. The higher the CW is, the higher the API is. For SC, we think with the fulfillment of relational psychological contract, it will have a sudden change above certain point. So we adopt step function to describe it. Considering DD falls into the range from 0 to 1 , to match the unit of OC, we use 10 multiply DD and plus 1 to describe OC. For equations see Appendix B.

(3) Changes of Relationship Justice (CRJ) Rate Variable InTree Branch. Changes of relational justice (CRJ) describe the changes in relational justice. According to Beuger and Baron (2001), it is jointly influenced by degree of distributive justice (DDJ), degree of procedural justice (DPJ), and degree of interactional justice (DIJ) [37]. Please see Figure 4. DDJ, DPJ, and DRJ are influenced by ATPCI and ARPCI, respectively, and, according to the reality, they are a little bit late compared to ATPCI or ARPCI. So for each of them, we apply delay function to describe. And to reduce the impact of unit on the values, for DIJ, we nest a log function into a delay function to reduce the value of FC multiplying ARPCI. For equations see Appendix B.

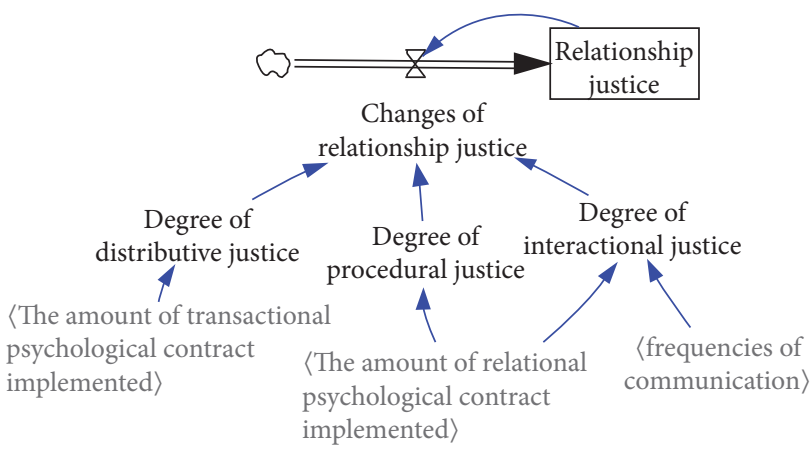

FIGURE 4: Changes of relationship justice (CRJ) rate variable in-tree branch.

(4) Changes of Transactional Psychological Contract Implemented (CTPCI) Rate Variable In-Tree Branch. Changes of transactional psychological contract implemented (CTPCI) describe the changes of fulfilled transactional obligations. According to the results of the above interviews, it is influenced by enterprise competency (EC) and degree of contract details (DCD). Please see Figure 5. EC provides an organization the potential and capability to fulfill the perceived transactional obligations. As an external variable, we consider the enterprise's condition and give the value to it. DCD decides the number of perceived transactional obligations in psychological contract. So CTPCI is jointly influenced by both of them. In terms of DCD, according to the above analysis, IORs can influence DCD. And we use 100 to divide the aggregation of the three aspects of IORs to describe DCD. For equations see Appendix B.

(5) Changes of Relational Psychological Contract Implemented (CRPCI) Rate Variable In-Tree Branch. Changes of relational psychological contract implemented (CRPCI) describe the changes of fulfilled relational obligations. According to the 


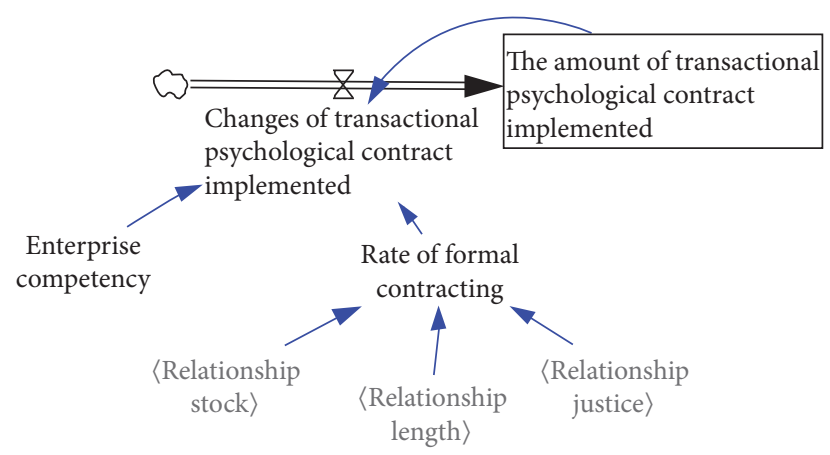

FIgURE 5: Changes of transactional psychological contract implemented (CTPCI) rate variable in-tree branch.

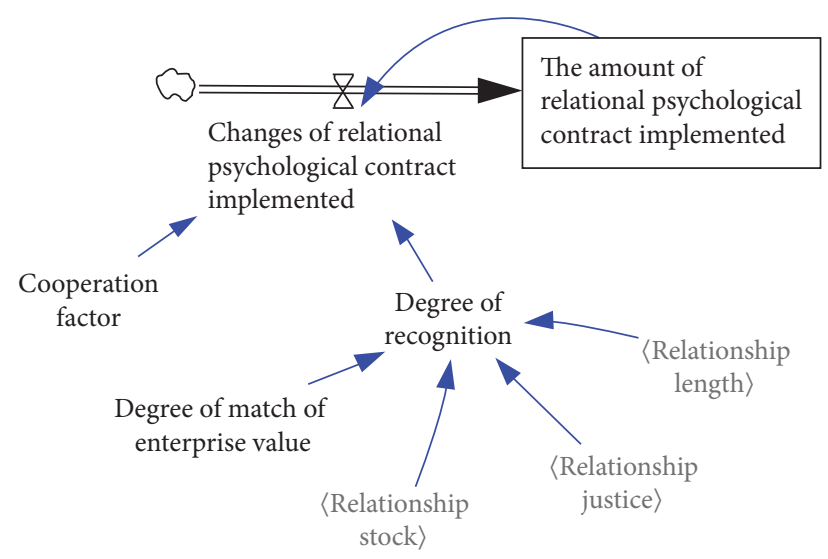

FIGURE 6: Changes of relational psychological contract implemented (CRPCI) rate variable in-tree branch.

results of the above interviews, it is mainly influenced by degree of recognition (DR) and cooperative factor (CF). Please see Figure 6. CF is the rate of cooperative willingness. Generally speaking, an organization is neutral to cooperation. So we suppose there is 0.5 possibilities for it to cooperate and thereby suppose CF is a constant and its value is 0.5 . DR is decided by two factors: the match degree of enterprise values (MDEV) and IORs. The higher MDEV is, the higher $\mathrm{DR}$ is. The more IORs are, the higher DR is. And we suppose $\mathrm{MDEV}$ is a constant and its value is 0.5 . For equations see Appendix B.

\subsection{Data Collection}

4.2.1. Data Collection. In this part, we take Jiangxi Motors Co., Ltd., as example to collect data and explore the interaction between IPC and IOR.

Jiangling Motors Co., Ltd. (JMC in abbreviation hereinafter), is a key player in China automotive industry. Its main product is commercial vehicles. Established in 1968, JMC develops quickly for the last 30 years. It went public in 1993 and has been ranked as one of China Top 100 Listed Companies for many years. Currently it has 15326 employees. In 2015 JMC sold 257016 vehicles and realized sales revenue of 24.53 billion RMB and volume.
In 1995, JMC introduced Ford Motor Company as strategic partner. Until now Ford company is the second biggest shareholder of JMC. As an early company which introduced foreign investment in Jiangxi province, with the support of its strategic partner-Ford Motor Company, Jiangling Motor Co., Ltd., develops rapidly. In 1997 JMC/Ford launched the first Sino-foreign jointly developed automobiles-Transit Light Bus. Thanks to its outstanding quality, Transit vehicles won the Ford Global Customer Satisfaction Gold Award for three consecutive years and were awarded as the best commercial vehicles in China in 2006 and 2007. With the cooperation with Ford, JMC has the opportunity to absorb the world's most advanced product technology, learn the manufacturing processes and management concepts of Ford company, and improve itself. Hence, from the above description, we can know the cooperation relationship of JMC and Ford company is one of IORs described by Oliver (1990) [4]. And the IOR of JMC and Ford is successful. The cooperation between them is regarded as a role model in auto industry in China and the long-term interorganizational relationship between them offers us lens to study the development of interorganizational relationship in Chinese context. Considering this, this paper choose the IOR of JMC and Ford company as example and we will further discuss how IPC coevolve with the development of IOR of the two companies.

In the system dynamics model, there are four external variables. In terms of degree of trust (DT), we use questionnaire to collect data. We adopt Zaheer et al. (1997) scale for interorganizational trust and invite three directors who directly interact with Ford Motors to fill this questionnaire [38]. All these directors are old employees in JMC, who have been working in JMC and interacting with Ford Motors for more than ten years. They are quite familiar with JMC's relationship with Ford Motors. One of them is from R\&D department. One of them is from production department, and one of them is from sales department. So they are asked to fill the questionnaire yearly. The questionnaire we use is Likert scale, which is the most widely used approach to scaling responses in survey research. When responding to a Likert item, respondents specify their level of agreement or disagreement on a symmetric agree-disagree scale for a series of statements. Thus, the range captures the intensity of their feelings for a given item. Then item responses are summed to create a score for a group of items. In this way we can quantify the abstract concepts in this paper. As such, we sum each director's response to all the items and get the score of each director for the degree of trust. Considering the responses of the three directors are of the same importance, we calculate the average value of the scores and then get the final score for degree of trust yearly. In terms of frequencies of communication (FC) and frequencies of transactions (FT), to avoid the biases deriving from the same persons who score the degree of trust, the persons who we invite to report the frequencies of communication and frequencies of transactions with Ford company are a little bit different. They are a director of $\mathrm{R} \& \mathrm{D}$ department., an executive vice-president who is responsible for the cooperation with Ford Motors, and an employee who has been working with Ford Motors since 1995, the year of which the two companies established the 
TABLE 2: Initial values of parameters.

\begin{tabular}{lccc}
\hline Parameter & Initial value & Parameter & Initial value \\
\hline ATPCI & 15 & Length of simulation & 15 \\
ARPCI & 13 & Unit & Year \\
RS & 8 & Step & 1 \\
RL & 7 & & \\
RJ & 7 & & \\
\hline
\end{tabular}

cooperation relationship. Considering the opinion of each of them is important, the values they reported are then averaged. In terms of enterprise competency (EC), we use business profits of JMC to represent enterprise competency.

The length of simulation is set to 180 months. The simulations were run from year 2005 to 2019. Table 2 shows a list of a parameters and initial values used in calibrating the model.

Considering most variables in this paper are abstract, we use relative values to represent each variable. The relationship between JMC and Ford company was established in 1995. Until 2005, it has developed for ten years. In such long term, both parties have accumulated certain amount of IORs. And at that time the psychological contract must be implemented more or less. To obtain the initial values of the five flow variables, we use questionnaires to collect the data. In terms of transactional psychological contract and relational psychological contract, we refer to Kingshott (2006) study [31]. In terms of relationship stock, relationship length, and relationship justice, we refer to $\mathrm{Wu}$ and Chen (2007) study [34]. For questionnaires please see Appendix A. We send these questionnaires to those department managers (both department managers and vice department managers) who have been working in JMC for more than ten years and ask them to fill the questionnaires according to the reality of 2005. Altogether there are 28 managers to fill these questionnaires. For each construct, we use Likert scale to measure it and the responses to items are summed to make the score for each construct. Then we sum the scores of all these people and then divide the number of these people to get the average value for each construct.

4.2.2. Validation. Model validation constitutes an important step in system dynamics methodology. Validity of the results of a given study is crucially dependent on the validity of the model. The validity of a model is not only helpful to improve the credibility of the model, but also contributing to building theories in the policy analysis. However, in this paper, because both IPCs and IORs are abstract concepts and in the actual environment we can not find comprehensive and accurate statistical data for these parameters, it is hard to have a comparison with the real conditions. In this case, based on the simulation results, we compare the development trends of the five level variables from 2005 to 2015 with the real development of IOR and IPC of JMC and Ford company to approximately test the evolution pattern of the model. In fact, this does not violate the original intention of SD simulation nor affect the discovery of the coevolution rule of IPCs and IORs [31]. In next part, we report the simulation results and

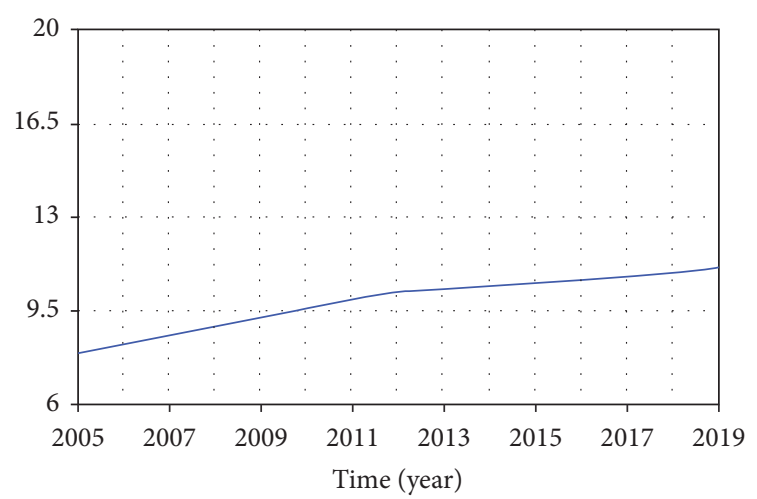

Relationship stock: — Current

FIGURE 7: Relationship stock (RS) trends.

analyze the results. From the analysis, the validity of the model is also illustrated.

\subsection{Simulation}

4.3.1. Simulation Results. There are 5 stock variables: relationship stock (RS), relationship length (RL), relationship justice (RJ), amount of transactional psychological contract implemented (ATPCI), and amount of relational psychological contract implemented (ARPCI). The simulation results of the five variables are shown below.

(1) Relationship Stock (RS) Variable. The simulation result is as shown in Figure 7.

From Figure 7, we can find that the RS increases with time, which indicates time span can affect RS. But the increase rate of RS is reduced. In 2005 both sides have already had some connections and relations. And this means after 10 years of cooperation both parties have accumulated certain amount of relationships. So the relationship stock is not starting from 0 . With time going by and more communications and cooperation, the relationship increases but with reduced growth rate. We can see at the beginning it develops faster. In 2005, JMC and Ford company have resigned the agreement on strategic relationship. Since 2005, their relationship enters in a new development stage. So after 2005 the relationship keeps increasing. But the growth rate is reduced. It shows with the link becoming stronger, the relationship accumulated per unit is reduced.

(2) Relationship Length (RL) Variable. The simulation result is as shown in Figure 8.

From Figure 8, we can see RL has not changed much during the simulation period. It keeps a small increase from 2005 to 2019. This matches the fact that the relationship is supposed to last long. This is because at the very beginning of the relationship, it gained great support of Jiangxi local government. With the support of government, the relationship is expected to last long. The development trend of RL well illustrates the anticipation. 


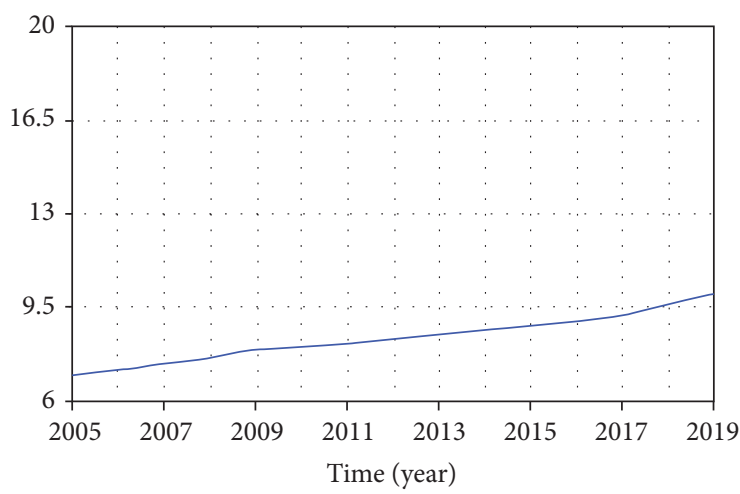

Relationship length:

— Current

FIGURE 8: Relationship length (RL) trends.

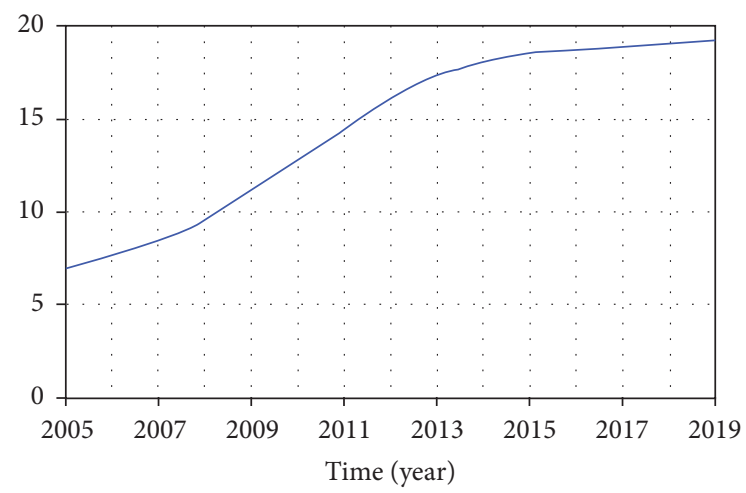

Relationship justice:

— Current

FIgURE 9: Relationship justice (RJ) trends.

(3) Relationship Justice (RJ) Variable. The simulation results are as shown in Figure 9.

From Figure 9, we can see that from 2005 to 2015 the relationship justice increases with relatively high speed comparing with the rate from 2015 to 2019. It indicates until 2015, through the cooperation, the relationship becomes more and more equal. RJ is increasing. After 2015, it also increases but with a little bit low rate. In fact, during the cooperation, with more knowledge transferred and learned by the relative weak side, the relationship tends to be symmetric. Only the increasing rate is reduced.

Figures 7, 8, and 9 not only can well explain the development trend of RS, RL, and RJ, it also shows the impact of IPC on IOR. With the implementation of IPC, the three dimensions of IOR all increase. Comparatively the influence of IPC on RL is not as significant as it is on RS and RJ.

(4) Amount of Transactional Psychological Contract Implemented (ATPCI) Variable. The simulation results are as shown in Figure 10.

From Figure 10, we can find that ATPCI is reduced with time. But there are no big changes of ATPCI. It decreases a little bit as time goes by. This coincides with our analysis of the

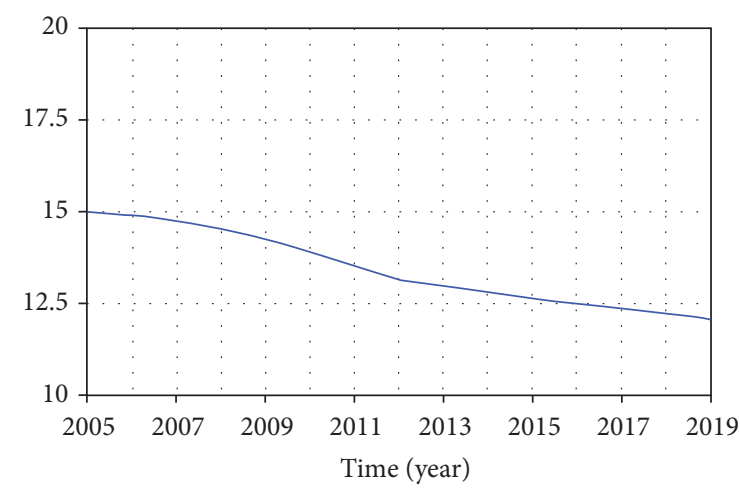

TPC:

- Current

FIGURE 10: Trends for amount of transactional psychological contract implemented (ATPCI).

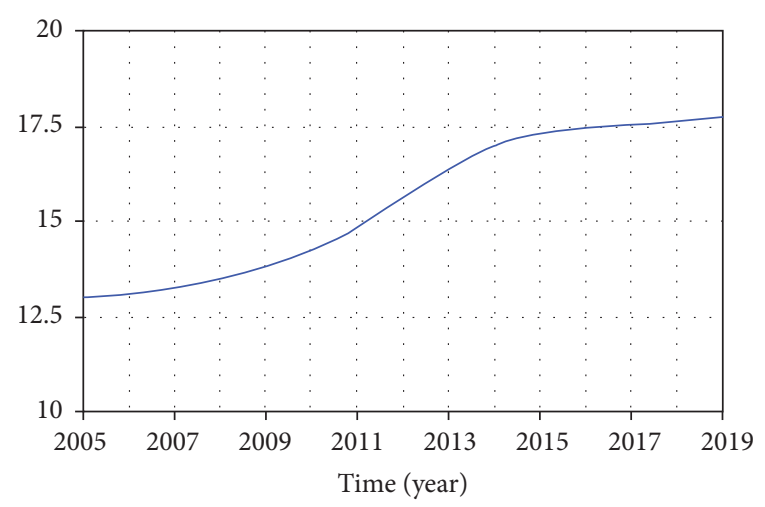

RPC:

- Current

FIGURE 11: Trends for amount of relational psychological contract implemented (ARPCI).

influence of IORs on IPCs. With more cooperation and the development of IORs, both JMC and Ford company intend to use relational norms rather than formal contracts to govern their cooperation. With the development of the relationship, the perceived transactional obligations are reduced a little bit mainly due to the less use of the formal contracts.

(5) Amount of Relational Psychological Contract Implemented (ARPCI) Variable. The simulation result is as shown in Figure 11.

From Figure 11, we can see that, at the first ten years, ARPCI increases with increased rate and at relatively high level. At the late 5 years, ARPCI increases with reduced rate and at low level. The result is very interesting. At the first ten years, with the increase of cooperation and the mutual understandings, both sides share values and missions with each other more. During this process, both sides are able to perceive more relational obligations with regard to their transactions. And with the development of IORs, they are more willing to fulfill these intangible, relational obligations such as being honest to their partner and trusting their partners. So at this stage, ARPCI increases quickly. However, later 


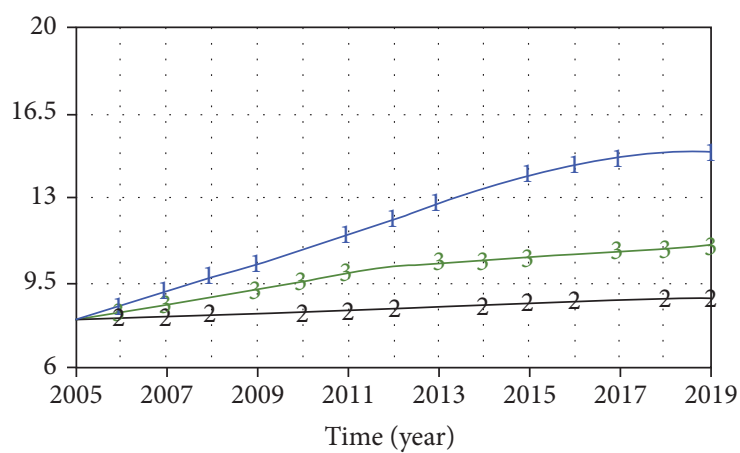

Relationship stock:

1 High

2 Low

3 Current

FIGURE 12: Influences of various trusts on relationship stock (RS).

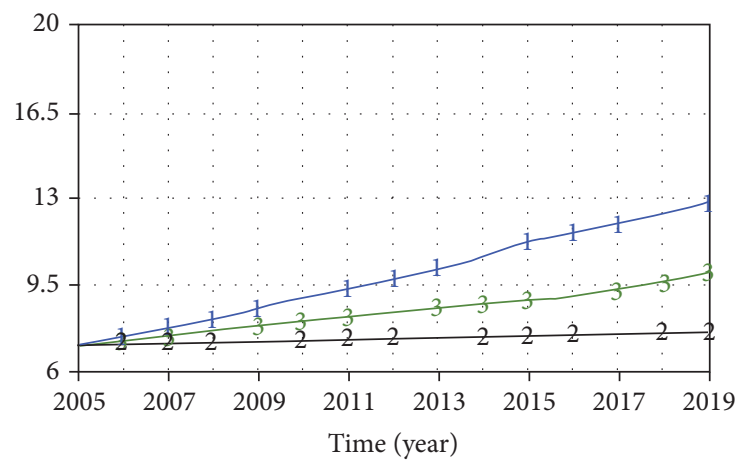

Relationship length:

1 High

2 Low

3 - Current

FIGURE 13: Influences of various trusts on relationship length (RL).

the perceived relational obligations gradually approach the highest point because through almost 10 years of cooperation both sides have already understood well what the other side wants in terms of their relational obligations. So the increased space is very little and the growth rate is reduced a lot.

4.3.2. Sensitivity Analysis. In this section, we adjust one important external variable to see its influence on IORs and IPCs. It is degree of trust (DT).

This study discusses the trends of IORs and IPCs from three trust scenarios by fixing other parameters: high degree of trust, medium degree of trust, and low degree of trust. High degree of trust is to be given relatively high points to degree of trust, whose value is $50 \%$ higher than the medium degree of trust. Low degree of trust is 50\% lower than the medium degree of trust. And the medium degree of trust is 15 . See from Figures 12 and 13, the higher the degree of trust is, the higher the relationship stock (RS) and relationship length (RL) are. It indicates that with high degree of trust JMC has accumulated more relationship and also has longer relationship. But Figure 14 shows another picture. When trust is high, relationship

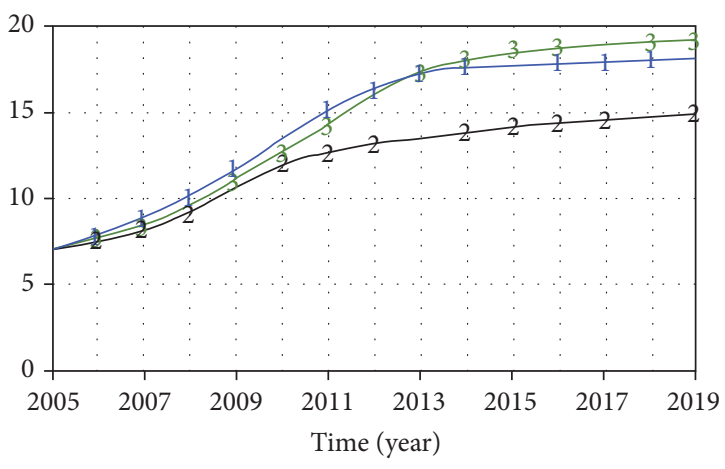

Relationship justice:

1 High

2 Low

3- Current

FIGURE 14: Influences of various trusts on relationship justice (RJ).

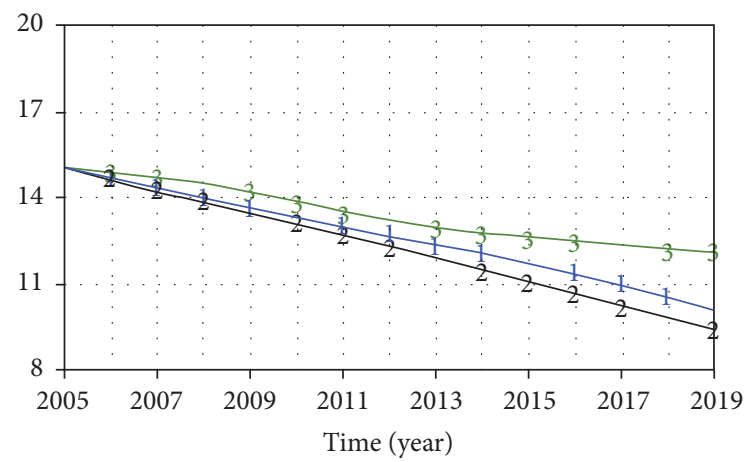

TPC:

1 High

2 Low

3- Current

FIGURE 15: Influences of various trusts on amount of transactional psychological contract implemented (ATPCI).

justice (RJ) increases rapidly and gets to its peak earlier than the other two scenarios. But its peak is between that of medium trust and low trust. For both medium and low degree of trust, they increase comparatively slow and it takes longer time for them to reach the peak point. But the medium degree of trust has the highest peak within the three scenarios. The result indicates that, at the beginning of an IOR, if the trust is high, then both sides can sense relatively high fairness in the relationship. And the perception of fairness increases quickly with time. However, in the high degree of trust scenario, the relationship justice is not the highest. So another implication of the result is that it is the medium degree of trust and not very high degree of trust that is the best conditions for JMC to achieve high relationship justice.

Figures 15 and 16 show the influence of trust on transactional psychological contract and relationship psychological contract, respectively. See from Figure 15, in the three scenarios the amount of transactional psychological contract implemented (ATPCI) changes very little with time. As the transactional obligations are mainly defined by formal 


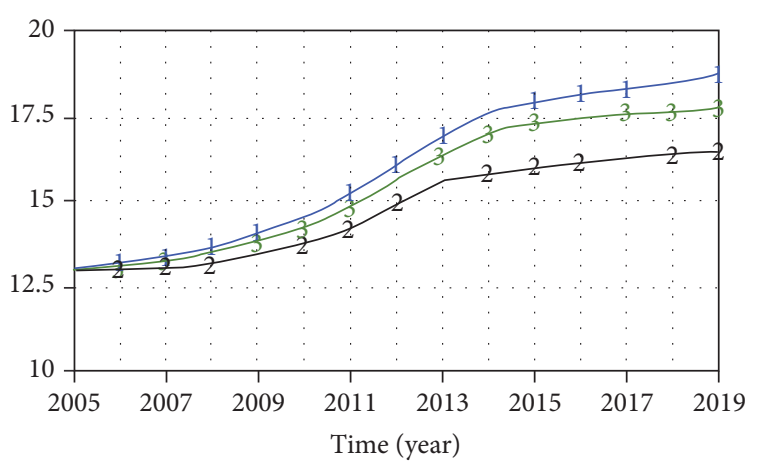

RPC:

1 High

2 Low

3 - Current

FIGURE 16: Influences of various trusts on amount of relational psychological contract implemented (ARPCI).

contracts and signing formal contracts is the indispensable process for an enterprise to cooperate, the amount of transactional obligations change very little with the development of relationship. But the medium degree of trust has the highest ATPCI and the low degree of trust has the lowest ATPCI. It indicates the medium degree of trust is the best condition for JMC to perceive and fulfill the transactional obligations in the cooperation. See from Figure 16, the higher the degree of trust is, the higher the amount of relational psychological contract implemented (ARPCI) is. The result supports the claim that the fulfillment of intangible and relational obligations in the cooperation relationship is closely related to trust between the two sides.

4.3.3. Summary and Discussions. Despite the common knowledge that IPCs and IORs are closely related, based on the structure analysis of their relationship, we can find that the interplaying between them is complex. Theoretically, IPCs mainly work through cooperation satisfaction to positively influence IORs while it mainly work through dependence and opportunistic behaviors to negatively influence IORs. Similarly, IORs also have both positive and negative influence on IPCs either through recognition or degree of contract details. The simulation results further test the analysis. The results reveal that relationship stock (RS), relationship length (RL), and relationship justice (RJ) develop with time and the fulfillment of IPCs. The total effect of IPCs on IORs is positive but the increase patterns of RS, RL, and RJ are different. However, in terms of the influence of IORs on IPCs, we need to differentiate the situations. For relational psychological contract, the effect of IORs is positive. It keeps in increase with the development of IORs. Nevertheless, during the same process, the fulfillment of transactional psychological contract almost keeps the same. It is probably because the negative influence of IORs offsets the positive influence. This finding reveals in detail the specific interactive influence between IPC and IOR and provides the development trend of both IPC and IOR. It contributes to deepening our understanding of the interplaying of IPC and IOR.
Based on the sensitivity analysis, we can further see the influence of trust on IORs and IPCs. In terms of the influence of trust, the higher the trust is, the higher the RS, RL, and ARPCI are. But for RJ and ATPCI, when trust is medium, we have the highest RJ and ARPCI. For perceived transactional obligations, each party understands them mainly based on legal contracts and explicit agreements. The present study reveals that the relational elements such as trust are not positively related to the fulfillment of these obligations. When trust is medium, both parties of a relationship can better communicate with each other especially about the economic elements in contract and will reduce the use of relational governance. All these contribute to the fulfillment of transactional obligations. At the same time, when trust is medium, the cooperative enterprises need not worry to be locked in their relationship and passively accept the relationship. So it is easier for enterprises to have the highest relationship justice when trust is medium. In a word, trust can exert great influence on both IORs and IPCs. But specifically, the influence of trust on RS, RL, and ARPCI is positive while the influence of trust on RJ and ATPCI is relatively complicated.

\section{Conclusions}

The coevolution of IORs and IPCs is an interesting topic, which contributes to our understanding of the development of IORs. In this paper, we build a SD model to simulate and analyze the coevolution of IORs and IPCs. Although this paper only use one company's data to simulate all the influences, it provides explorative and empirical evidence on the relationship between IPC and IOR as well as the relationship between trust and IPC and IOR. It further reveals how IPCs interplay with IOR and how trust impacts IPC and IOR in detail, which from quantitative perspective enriches our knowledge about how IOR develops.

Based on the results, some managerial implications can be drawn. The influence of IPCs on IORs is positive, which indicates more attentions and importance should be placed on the management of expectations of enterprises. Well understanding what obligations are expected from the partners matters much for the development of IORs. So we need to understand and more importantly constantly fulfill the obligations expected from our cooperative enterprises. In particular, we should attach importance to the intangible and relational obligations. From the results we can find IORs have positive influence on ARPCI but can hardly influence the changes of ATPCI. It indicates that a good IOR can result in the fulfillment of relational psychological contract. In conjunction with the influence of IPCs on IORs, we can find IORs and relational psychological contract form a benign circle. This finding sharpens our understanding about the relationship between IORs and IPCs and reminds us with the importance of relational psychological contract. For most exchanges, organizations often place much emphasis on the fulfillment of the tangible and economic obligations and ignore the intangible and relational obligations. This finding shows us that the relational psychological contract is an important determinant for the development of IORs. 
Secondly, according to the results, trust has impact on IORs and IPCs. This finding indicates that trust is a determinant for both IORs and IPCs. Therefore, it is important to build trust between cooperative enterprises. For example, enterprises can establish trust by active communications. Active and open exchange of information is the intrinsic meaning of trust and also a very effective path to build trust [31]. Communication not only provides sufficient evidence for partner enterprise that it is trusted but also provides channel to prove itself can be trusted. What is more, it helps to eliminate the misunderstandings arising from unfamiliarity and poor information [34]. Another important point enterprises should know about trust is that too high trust sometimes is not good for both IOR and IPC. As discussed by some papers, too high trust may bring some problems for enterprises [39]. As revealed in this study, medium level trust is good for relationship justice and fulfillment of transactional psychological contract. So this reminds us when building trust between organizations, we should obey the "moderate principle." At the same time, enterprises should adjust dynamically in accordance with the changes of its partner enterprise either concerning the building of trust or the development of IORs. Without dynamic adjustment, IORs can not be developed and enhanced even with abundant transactions.

However, the study also has some limitations. Because of the obtainability of data and the abstraction of variables, although the model is built on careful theoretical analysis and investigation, it will make the study more thorough if the model can be further tested by more cases. Future research could be developed in this direction.

\section{Conflicts of Interest}

The author declares no conflict of interests. The author has no financial and personal relationships with other people or organizations that can inappropriately influence the work.

\section{Acknowledgments}

This work was supported by the China Scholarship Council, the National Natural Science Foundations of China (Grant nos. 71462009 and 71361013), the Natural Science Foundations of Jiangxi Province (Grant no 20171BAA208013), and the Science and Technology Project of Jiangxi Provincial Department of Education (Grant no. GJJ160440).

\section{References}

[1] D. Lavie, "Alliance portfolios and firm performance: a study of value creation and appropriation in the U.S. software industry," Strategic Management Journal, vol. 28, no. 12, pp. 1187-1212, 2007.

[2] J. Hagedoorn, "Understanding the rationale of strategic technology partnering: Nterorganizational modes of cooperation and sectoral differences," Strategic Management Journal, vol. 14, no. 5, pp. 371-385, 1993.

[3] G. Sánchez-González and L. Herrera, "User cooperation effects on firm's innovation outputs," Canadian Journal of Administrative Sciences/Revue Canadienne des Sciences de l'Administration, vol. 32, no. 2, pp. 86-101, 2015.
[4] C. Oliver, "Determinants of Interorganizational Relationships: Integration and Future Directions.," Academy of Management Review, vol. 15, no. 2, pp. 241-265, 1990.

[5] A. Takeishi, "Bridging inter- and intra-firm boundaries: management of supplier involvement in automobile product development," Strategic Management Journal, vol. 22, no. 5, pp. 403433, 2001.

[6] R. D. Ireland, M. A. Hitt, and D. Vaidyanath, "Alliance Management as a Source of Competitive Advantage," Journal of Management, vol. 28, no. 3, pp. 413-446, 2002.

[7] G. Lorenzoni and A. Lipparini, "The leveraging of interfirm relationships as a distinctive organizational capability: a longitudinal study," Strategic Management Journal, vol. 20, no. 4, pp. 317-338, 1999.

[8] A. Parkhe, "Strategic alliance structuring: a game theoretic and transaction cost examination of interfirm cooperation," Academy of Management Journal, vol. 36, no. 4, pp. 794-829, 1993.

[9] W. He and S.-H. Chen, "Game analysis of determinants of stability of semiconductor modular production networks," Sustainability, vol. 6, no. 8, pp. 4772-4794, 2014.

[10] W. He, "China technology innovation strategy-from technology transfer perspective," Information-An International Interdisciplinary Journal, vol. 15, no. 11, pp. 4841-4846, 2012.

[11] S.-H. Chen, "The influencing factors of enterprise sustainable innovation: an empirical study," Sustainability, vol. 8, no. 5, article 425, 17 pages, 2016.

[12] B. L. Simonin, "The importance of collaborative know-how: an empirical test of the learning organization," Academy of Management Journal, vol. 40, no. 5, pp. 1150-1174, 1997.

[13] M. A. Hitt, M. T. Dacin, E. Levitas, J.-L. Arregle, and A. Borza, "Partner selection in emerging and developed market contexts: resource-based and organizational learning perspectives," Academy of Management Journal, vol. 43, no. 3, pp. 449-467, 2000.

[14] S. H. Chen, "Construction of an early risk warning model of organizational resilience: an empirical study based on samples of R \& D teams," Discrete Dynamics in Nature and Society, vol. 2016, Article ID 4602870, 9 pages, 2016.

[15] W. E. D. Creed and R. E. Miles, “Trust in organizations: a conceptual framework linking organizational forms, managerial philosophies, and the opportunity costs of controls," in Trust in Organizations: Frontiers of Theory, R. M. Kramer and T. R. Tyler, Eds., pp. 16-38, 1996.

[16] N. Anderson and R. Schalk, "The psychological contract in retrospect and prospect," Journal of Organizational Behavior, vol. 19, no. 1, pp. 637-647, 1998.

[17] R. F. Lusch and J. R. Brown, "Interdependency, Contracting, and Relational Behavior in Marketing Channels," Journal of Marketing, vol. 60, no. 4, pp. 19-38, 1996.

[18] M. V. Roehling, "The origins and early development of the psychological contract construct," Journal of Management History (Archive), vol. 3, no. 2, pp. 204-217, 1997.

[19] W. He, "An inventory controlled supply chain model based on improved BP neural network," Discrete Dynamics in Nature and Society, vol. 2013, Article ID 537675, 7 pages, 2013.

[20] S.-H. Chen and W. He, "Study on knowledge propagation in complex networks based on preferences, taking wechat as example," Abstract and Applied Analysis, vol. 2014, Article ID 543734, 11 pages, 2014.

[21] S. L. Robinson and D. M. Rousseau, "Violating the psychological contract: Not the exception but the norm," Journal of Organizational Behavior, vol. 15, no. 3, pp. 245-259, 1994. 
[22] C. Grönroos, "Relationship approach to marketing in service contexts: The marketing and organizational behavior interface," Journal of Business Research, vol. 20, no. 1, pp. 3-11, 1990.

[23] A. W. Gouldner, "The norm of reciprocity: a preliminary statement," American Sociological Review, vol. 25, no. 2, pp. 161-178, 1960.

[24] R. P. J. Kingshott, "Psychological contracts within supplier-distributor relationships," in Proceedings of the IMP Conference, Perth, Australia, 2002.

[25] W. H. Gao and X. K. Chen, "Interorganization relationship: explanation based on psychological contract," Economic Management Journal, vol. 30, no. 21-22, pp. 101-107, 2008.

[26] S.-H. Chen, S.-H. Xu, C. Lee, N. N. Xiong, and W. He, “The study on stage financing model of IT project investment," The Scientific World Journal, vol. 2014, Article ID 321710, 6 pages, 2014.

[27] S.-H. Chen, "The game analysis of negative externality of environmental logistics and governmental regulation," International Journal of Environment and Pollution, vol. 51, no. 3-4, pp. 143155, 2013.

[28] M. Pepur, Z. Mihanovi, and S. Pepur, "The impact of psychological contract on relationship quality in financial services market," Croatian Operational Research Review, no. 4, pp. 89-98, 2013.

[29] S. J. Zhai, P. Huang, and W. H. Gao, "A new research paradigm of inter-firm relationships: characterization of, expectation/obligation , through psychological contracts," Commercial Research, vol. no.3, pp. 115-124, 2014.

[30] J. W. Forrester, "Industrial dynamics: a major break through for decision makers," Harvard Business Review, vol. 36, no. 4, pp. 37-66, 1958.

[31] R. P. Kingshott, "The impact of psychological contracts upon trust and commitment within supplier-buyer relationships: a social exchange view," Industrial Marketing Management, vol. 35, no. 6, pp. 724-739, 2006.

[32] A. Valtakoski, "Initiation of buyer-seller relationships: The impact of intangibility, trust and mitigation strategies," Industrial Marketing Management, vol. 44, pp. 107-118, 2015.

[33] R. W. Palmatier, "Interfirm Relational Drivers of Customer Value," Journal of Marketing, vol. 72, no. 4, pp. 76-89, 2008.

[34] Z. W. Wu and Y. Chen, "The measurement and performance analysis of inter-firm relationship," Forecasting, no. 2, pp. 8-13, 2007.

[35] C. Zhang, T. Zhang, and G. J. Zhuang, "Impacts of channel tie strength on the channel power exercises: from the perspective of relational embeddedness," Journal of Management Science, vol. 25, no. 3, pp. 56-68, 2012.

[36] L. Ya, R. Li-rong, and L. Ye, “The influence of organizational justice on organizational outcome variables," Management World, vol. 3, pp. 6-13, 2003.

[37] C. D. Beuger and R. A. Baron, "Perceptions of systematic justice: the effects of distributive, procedural, and interactional justice," Journal of Applied Social Psychology, vol. 31, no. 2, pp. 324-339, 2001.

[38] A. Zaheer, B. McEvily, and V. Perrone, "Does Trust Matter? Exploring the Effects of Interorganizational and Interpersonal Trust on Performance," Organization Science, vol. 9, no. 2, pp. 141-159, 1998.

[39] Y. Liua, Y. Lib, L. Taoa, and Y. Wanga, "Relationship stability, trust and relational risk in marketing channels: evidence from China," Industrial Marketing Management, vol. 37, no. 4, pp. 432-446, 2008. 


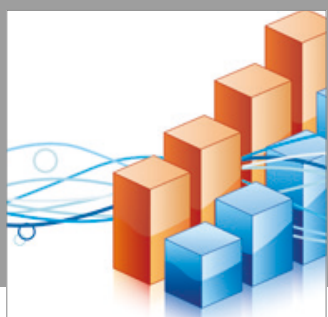

Advances in

Operations Research

vatersals

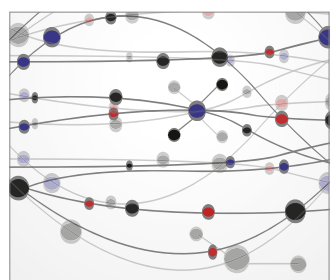

\section{The Scientific} World Journal
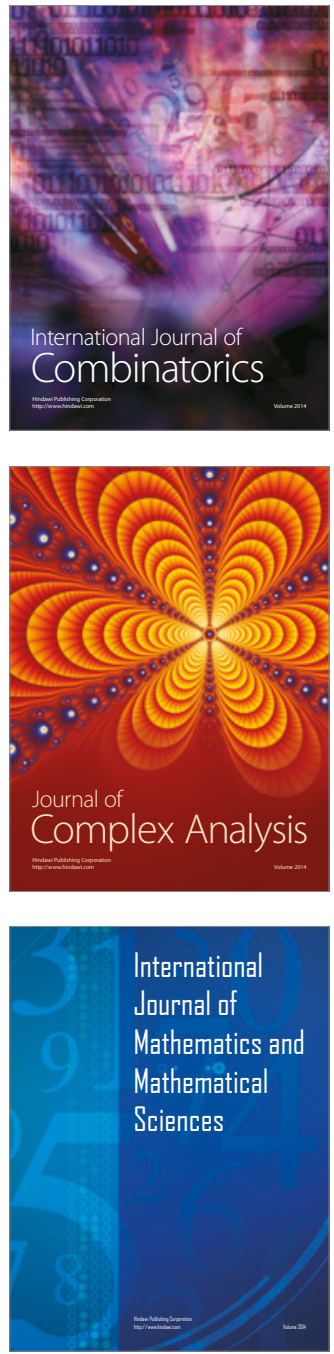
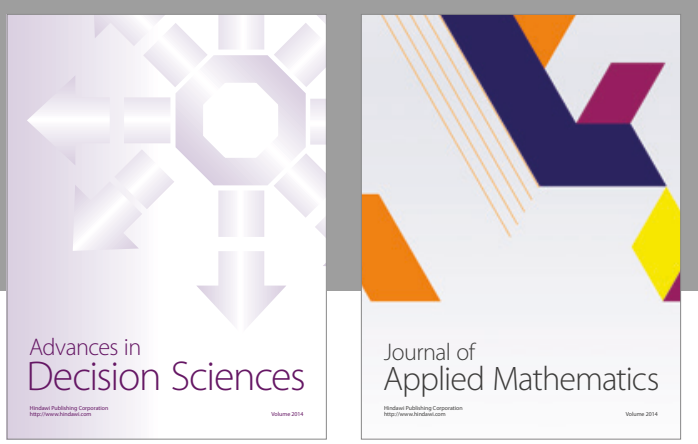

Algebra

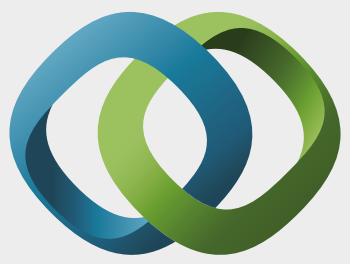

\section{Hindawi}

Submit your manuscripts at

https://www.hindawi.com
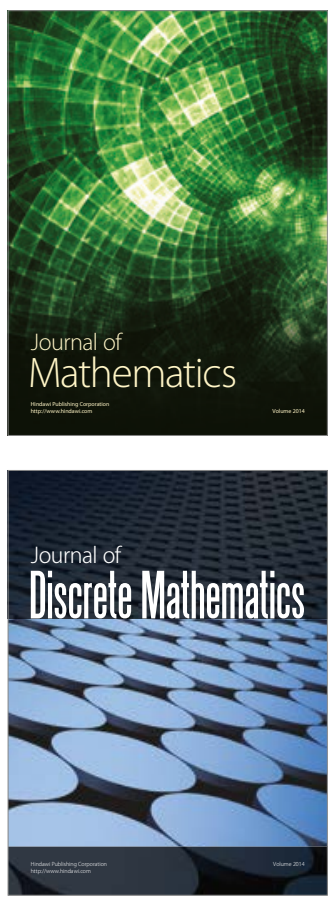

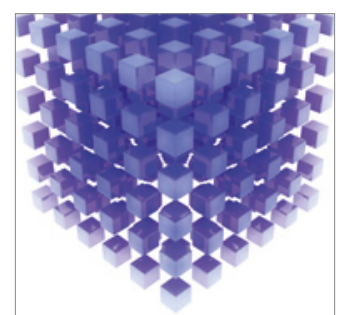

Mathematical Problems in Engineering
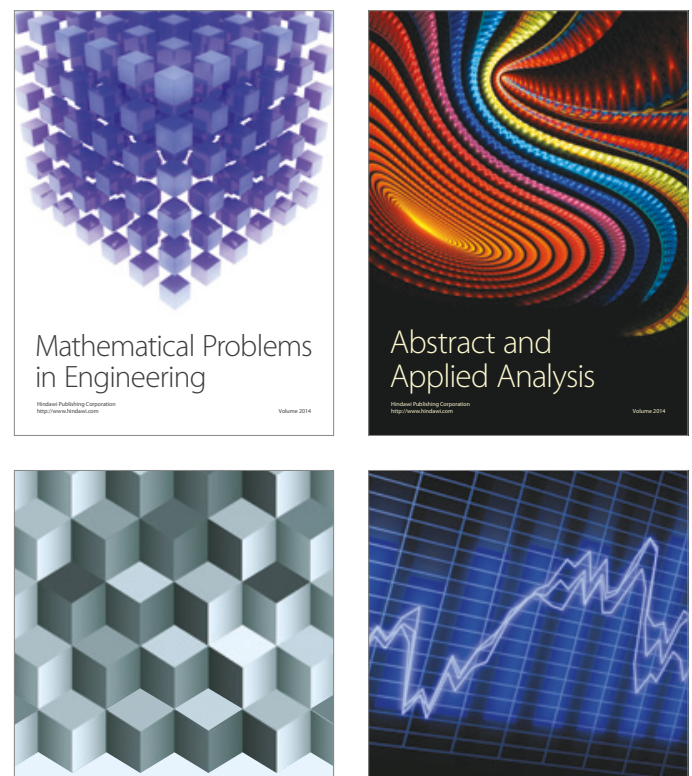

Journal of

Function Spaces

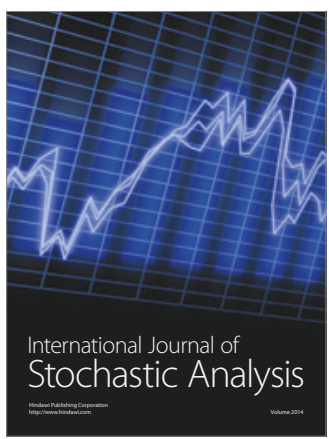

Probability and Statistics
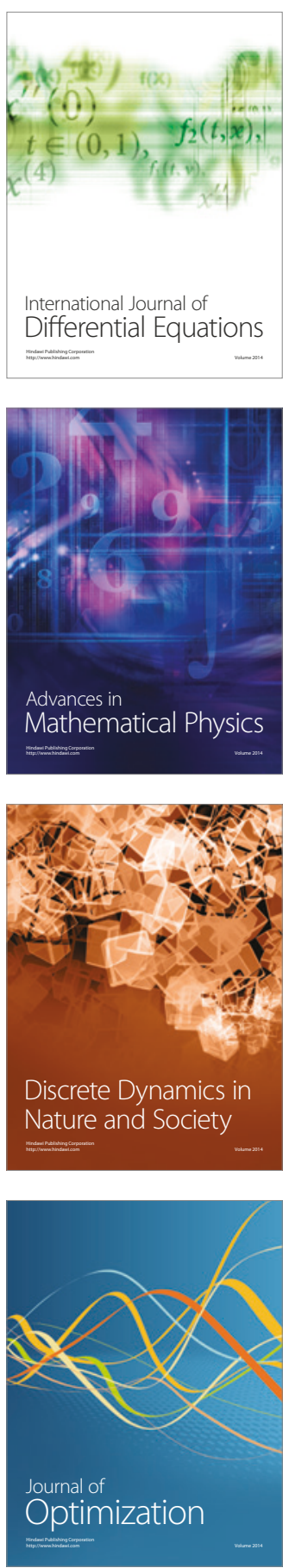